\title{
The use of geometric probability to change the probability of finding a series of random deposition coins Filatov $\mathbf{O}$. \\ Применение геометрической вероятности для изменения вероятности нахождения серий случайных выпадений монеты Филатов О. В.
}

Филатов Олег Владимирович / Filatov Oleg - инженер-программист, Научно-технический центр «Модуль», г. Москва

\begin{abstract}
Аннотация: исторически первой системой изменения вероятности обнаружения серий случайных событий была игра Уолтера Пенни. В статье показано, что вероятность обнаружения серий случайных бинарных событий фиксированной длины зависит от способов их поиска в последовательности. Для изменения вероятности обнаружения выпавщих серий случайных бинарных событий применена геометрическая вероятность.

Abstract: historically, the first system to change the probability of detecting a series of random events, the game was Walter Penny. The article shows that the probability of detection of a series of random binary events fixed length depends on how they search in the sequence. Geometric probability applied to change the probability of detection of the separated random series of binary events.
\end{abstract}

Ключевые слова: игра Пенни, Рихард Мизес, система игры, выпадения монеты, потоковая последовательность, коллектив, элементарное событие, эл, составные события, составное событие, потоковая вероятность, геометрическая вероятность.

Keywords: penny game, Richard von Mises, game system, loss of coins, threading sequence, staff, elementary event, el, composite events, a composite event streaming probability, geometric probability.

\section{Сокращения и термины:}

эл - элементарное событие, элементарный эксперимент типа броска монеты, эл является футляром для хранения результат «броска монеты» («0», «1»);

пос-ть - последовательность.

\section{Введение}

Рассмотрим последовательную запись результатов случайных выпадений монеты: «0100110111110100000011101...», где «1» и «0» обозначение сторон монеты. Серии одинаковых событий можно представить в виде отрезков, длина которых определяется числом выпавших подряд одинаковых событий («0» или «1»).

Примеры. Пять выпавших единиц «1111 » есть отрезок длиной пять. Шесть нулей « $\underline{\mathbf{0 0 0 0 0 0}}$ ” есть отрезок длины шесть. Нуль «ㅃ», перед которым и за которым находятся единицы «1», назовём отрезком единичной длины.

Разделив на отрезки последовательную запись случайных выпадений монеты, мы создали условия для применения геометрической вероятности.

\section{Определение геометрической вероятности}

Геометрической вероятностью называется вероятность попадания точки в пространственную область пропорционально размеру этой области (длине, площади).

Опишем подробнее объект исследования. Случайные результаты выпадения монеты обозначим на оси через ноль «0» и единицу «1» в порядке их выпадения. Каждое случайное событие представим на оси в виде отрезка, который будем называть элементарным (элом). Размер всех эл одинаков и не зависит от хранящегося в нём результата выпадения монеты («0, «1»). В любой момент времени число элементарных отрезков на оси определено и равно N. Рихард Мизес назвал такие последовательности коллективами, но автору больше нравится название «Потоковая последовательность», которое лучше отражает зависимость числа $\mathrm{N}$ элементарных событий от времени. Будем обозначать потоковую последовательность большой буквой $\mathrm{F}$ - первой буквой английского слова flow (поток). Элементарные отрезки, содержащие одинаковые значения («0» или «1»), объединяются в отрезки случайных длин - составные события. Геометрическая вероятность попадания в составные события зависит от их длин.

Организуем перемещение некоего абстрактного устройства над осью. Из устройства, как этого требует определение геометрической вероятности, на ось «падают» точки. Будем называть зондами эти безразмерные падающие точки. Потребуем, чтобы между двумя зондами было расстояние не менее 25 эл (при меньшей величине шага возможно многократное попадание нескольких зондов в один и тот же отрезок (составное событие)). Потребуем, чтобы устройство, перемещающееся над осью, не различало значения эл («0» или «1»), над которыми оно перемещается, но имело возможность знать количество пропущенных эл. По классической теории вероятностей знание числа пропущенных эл не поможет определить величину («0» или «1») зондируемого эла. 
Если выполняется всё выше описанное, то по определению геометрической вероятности растёт вероятность попадания в длинные отрезки и уменьшается вероятность попадания в короткие.

Результатом зондирования отрезков (составных событий) точками является изменение спектра длин отрезков, по сравнению со спектром длин, полученным при последовательном определении длин отрезков оси. Более вероятное попадание точек в длинные отрезки приводит к процентному росту обнаруженных длинных отрезков и сокращению процента обнаруженных коротких отрезков.

Основная часть

Проведём экспериментальное изучение эффектов геометрической вероятности, исследуя распределение численности отрезков разных длин, в которые попадают зонды. Для этого последовательность, полученную от физического генератора случайных бинарных событий, запишем в компьютерный файл. С помощью программы, производящей погружения зонда $\mathrm{Z}_{\mathrm{G}}$ в файл на каждый $\mathrm{k}=25$ эл, определяем длины отрезков ${ }^{\mathrm{n}} \mathrm{S}$, в которые попадал зонд $[2,5]$. Разделив число обнаруженных отрезков ${ }^{\mathrm{n}} \mathrm{S}$ на число всех зондирований, получим $\mathrm{P}_{\mathrm{G}}$ - геометрическую вероятность попадания зондом $\mathrm{Z}_{\mathrm{G}}$ в отрезок (составное событие) ${ }^{\mathrm{n}} \mathrm{S}$, длиной $\mathrm{n}$ эл. На графике 1 кривая геометрической вероятности $\mathrm{P}_{\mathrm{G}}$ обозначена «Геометр-кая вер-ть».

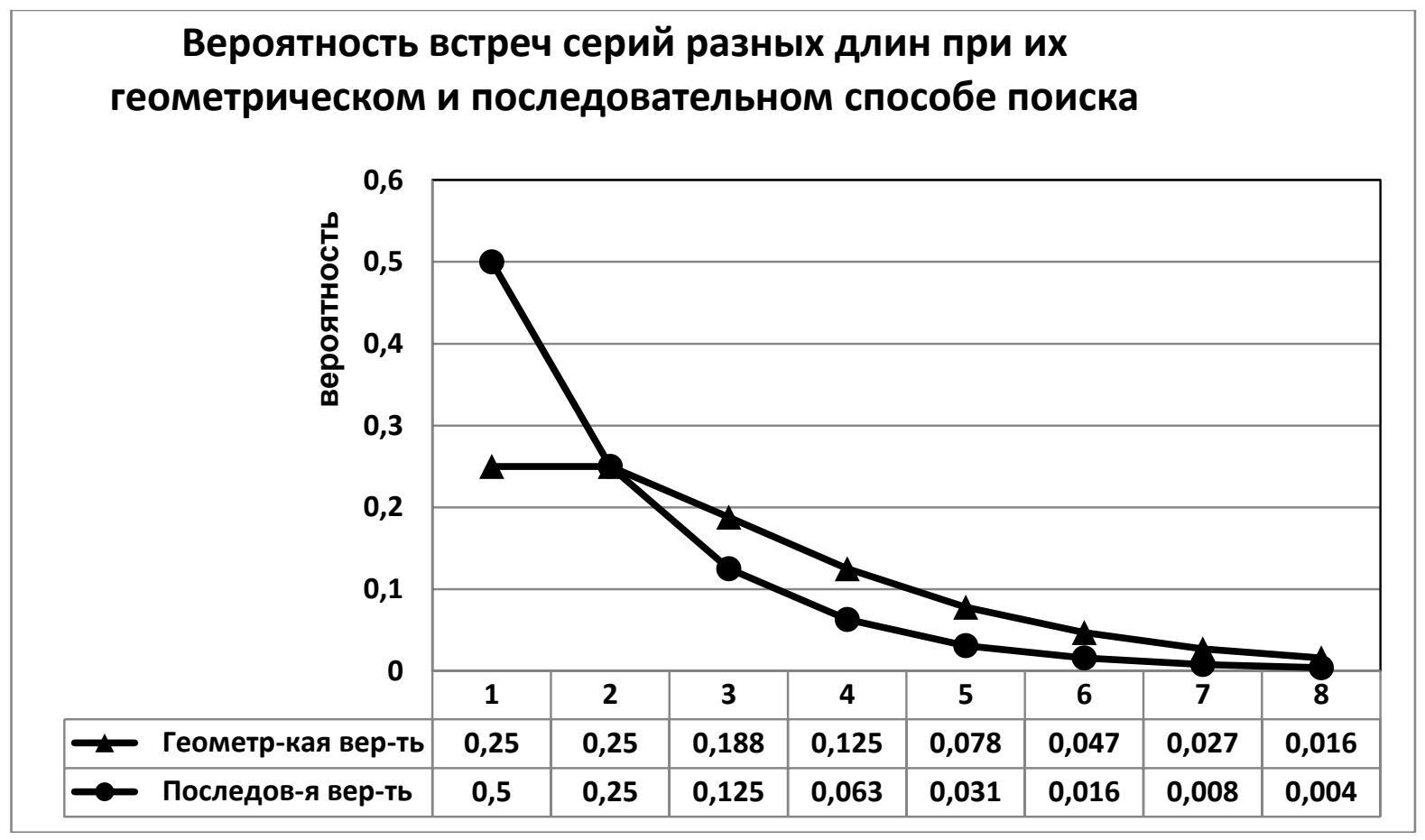

График 1. Вероятность встреч разных длин при их геометрическом и последовательном способе поиска 5]:

Формула 1.1 описывает геометрическую вероятность ${ }^{n} p_{\mathrm{g}}$ внедрения зонда в отрезки разной длины $n[2$,

$$
{ }^{n} p_{\mathrm{g}}=\frac{n}{2^{n+1}}
$$

Формула 1.2 позволяет рассчитать математическое ожидание числа отрезков ${ }^{n} S_{\mathrm{g}}$ длины n, полученных при использовании геометрического (пространственного) эффекта, в потоковой по-ти из $N$ элементарных событий, с пропуском (шагом) между зондовыми замерами $\mathrm{k}$ эл [2, 5]:

$$
{ }^{n} S_{\mathrm{g}}=\frac{N}{k} \cdot \frac{n}{2^{n+1}}=Z_{g} \cdot \frac{n}{2^{n+1}}
$$

Число элементарных событий $N$ потоковой последовательности связано через пропуск (измеряемый в элах шаг) k между зондовыми замерами, с числом зондирований $Z_{g}$, по ф. 1.3:

$$
Z_{\mathrm{g}}=\frac{N}{k}
$$


Поскольку в статье показывается, что вероятность обнаружения случайных бинарных отрезков зависит от способа их поиска, то опишем способ поиска отрезков в потоковой последовательности $\mathrm{F}$, отличный от геометрического, который назовём «Потоковым». А вероятность нахождения отрезков «Потоковым» способом обозначим: ${ }^{n} P_{F}[1,2,3,4]$. На графике 1 потоковая вероятность обозначена «Последов-я вер-ть».

Описание потокового способа поиска составных событий в $F$. С помощью компьютерной программы последовательного поиска найдём распределение численности отрезков разных длин [2, 3]. Программа выявляет последовательно, друг за другом, в очерёдности, существующие в файле отрезки всех длин. Программа поочерёдно считывает значения элов (элементарных событий) из файла и определяет длину отрезка, образуемого одинаковыми элами. Каждый найденный отрезок увеличивает счётчик отрезков для отрезка (составного события) данной длины. Деля число отрезков ${ }^{n} S_{F}$ длины n на число всех отрезков $S_{F}$ бинарной потоковой пос-ти $\mathrm{F} 0,5(\mathrm{~N})$ получим потоковую вероятность ${ }^{n} P_{F}$ выпадения отрезка длины $\mathrm{n}$ ф. 2.1 $[1,2,3,4]$ :

$$
{ }^{n} P_{F}=\frac{{ }^{n} S_{F}}{S_{F}}=\frac{1}{2^{n}}
$$

На графике 1 кривая потоковой вероятности ${ }^{n} P_{F}$ обозначена как «Последов-я вер-ть».

Определение потоковой вероятности. Привычную всем формулу вероятности $\frac{1}{2^{n}}$ можно получить как отношение числа составных событий ${ }^{n} S_{F}$ длины n ко всем составным событиям $S_{F}$ бинарной потоковой пости F0,5 (N), ф. 2.1. Такая трактовка вероятности: отношение составных событий ${ }^{n} S_{F}$ к общему числу составных событий $S_{F}$ потоковой пос-ти $\mathrm{F} 0,5(\mathrm{~N})$, является новым, самостоятельным определением вероятности, основанной на знании законов образования составными событиями случайной бинарной последовательности [2, 4].

Действительно, любая случайная бинарная пос-ть F0,5 (N) однозначно образуется из составных событий ${ }^{n} S_{F}[1,2,3,4]$, численность которых в пос-ти F0,5 (N) связана с числом элементарных событий $\mathrm{N}$ по ф. 2.2.

$$
{ }^{n} S_{F}=\frac{N}{2^{n+1}}
$$

Где: $\mathrm{N}$ - число событий в пос-ти.

Следствием теоремы «О амплитудно-частотной характеристике идеальной бинарной случайной последовательности» [2, 4] является то, что число всех отрезков $S_{F}$ в потоковой пос-ти с достаточно большим числом эл $\mathrm{N}$, будет динамически равно N/2, ф. 2. 3:

$$
S_{F}=\frac{N}{2}
$$

Исходя из ф. 2.2 и ф. 2.3 перепишем формулу потоковой вероятности 2.1 в виде ф. 2.4:

$$
{ }^{n} P_{F}=\frac{{ }^{n} S_{F}}{S_{F}}=\frac{N}{2^{n+1}}: \frac{N}{2}=\frac{1}{2^{n}}
$$

Обе вероятности: потоковая, ф. 2.4 и геометрическая, ф.1.1 - являются отношением выпавших серий ${ }^{n} S$ искомой длины n к полному числу серий всех длин $S$ (включая серии искомой длины).

Забегая вперёд, отметим, что потоковая вероятность вводилась для учёта распределения составных событий в бинарной последовательности, путём просмотра эл в порядке их поступления от генератора случайных событий, и формирования из них составных событий, без всяких предсказательных эффектов. Последовательная вероятность вводилась для алгоритмического исследования предсказательных эффектов при пос-ном просмотре эл друг за другом. Но так как формулы вероятностей для этих процессов одинаковы, ф. 2.4, ф. 3.2, то смысл этих двух названий вероятностей совпал в этой статье.

Для получения формулы потоковой вероятности ф. 2.4, было проведено деление числа составных событий ${ }^{n} S_{F}$ длины $n$ на число всех составных событий пос-ти $S_{F}$. Поэтому формально разделим число попаданий зонда в составные события ${ }^{n} S_{\mathrm{g}}$ длины $n$, ф. 1.2 на число всех составных событий пос-ти $S_{F}$, ф. 2.3 , и получим ${ }^{n} f_{\mathrm{g}}$ - относительную геометрическую частоту встреч составных событий длины $n$ в бинарной F0,5 (N) последовательности, ф. 2.5: 


$$
{ }^{n} f_{\mathrm{g}}=\frac{{ }^{n} S_{\mathrm{g}}}{S_{F}}=\frac{N}{k} \cdot \frac{n}{2^{n+1}}: \frac{N}{2}=\frac{1}{k} \cdot \frac{n}{2^{n}}
$$

Геометрическая природа процесса очевидно присутствует в относительной геометрической частоте встреч ${ }^{n} f_{\mathrm{g}}$ в виде множителя $n$, а бинарная вероятность представлена классическим множителем: $\frac{1}{2^{n}} .$. Геометрическая вероятность ${ }^{n} p_{\mathrm{g}}$, ф. 1.1 , пропорциональна ${ }^{n} f_{\mathrm{g}}$ с коэффициентом $\mathrm{g}=\frac{k}{2}$, то есть: ${ }^{n} p_{\mathrm{g}}=\mathrm{g} \cdot$ ${ }^{n} f_{\mathrm{g}}=\frac{k}{2} \cdot{ }^{n} f_{\mathrm{g}} \cdot$

Изучим потоковую и геометрическую вероятности через алгоритмы поиска серий из элементарных событий. Исследуем и сравним поисковую эффективность потоковой и геометрической вероятности между собой. Для этого уровняем количества попыток угадать серии заданной длины, для обоих способов поиска вероятностей ${ }^{n} f_{\mathrm{g}}$ и ${ }^{n} P_{F}$. То есть, каждым способом поиска (потоковым и геометрическим) будет сделано по $\mathrm{Z}$ попыток угадать серию из $\mathrm{n}$ элементарных событий (эл). Будем фиксировать не только числа угаданных и не угаданных серий, но и количества эл, задействованных в этих попытках, для каждого из способов.

Примеры поиска серий нужной длины обоими способами

Назовём геометрический способ поиска «Геометрическим алгоритмом» (ГА), а потоковый способ поиска «Последовательным алгоритмом» (ПА). Оба алгоритма предсказывают величины выпадающих элементарных событий и проверяют свои предсказания на истинность.

Приведём поясняющие примеры поиска серий нужной длины для обоих способов поиска вероятностей ${ }^{n} f_{\mathrm{g}}$ и ${ }^{n} P_{F}$, при помощи таблицы 1.

Таблица 1. Некоторые ситуаичи поискового алгоритма, $L=5$

\begin{tabular}{|c|c|c|c|c|c|c|c|c|c|c|c|}
\hline \multicolumn{6}{|c|}{ Последовательный (ПА) } & \multicolumn{6}{|c|}{ Геометрический (ГА) } \\
\hline 1 & 2 & 3 & 4 & 5 & 6 & 7 & 8 & 9 & $0^{1}$ & $1^{1}$ & $2^{1}$ \\
\hline & $(+)^{1}$ & $(+)^{2}$ & $(+)^{3}$ & $(+)^{4}$ & $(+)^{5}$ & $(+)^{4}$ & $(+)^{3}$ & $(+)^{2}$ & $(+)^{1}$ & & $(+)^{5}$ \\
\hline$=1$ & 1 & 1 & 1 & 1 & 0 & 0 & 1 & 1 & 1 & $=1^{Z}$ & 0 \\
\hline & ${ }_{(+)}^{1}$ & $(+)^{2}$ & $(+)^{3}$ & ${ }_{(+)}^{4}$ & $(-)^{5}$ & $(-)^{4}$ & $(+)^{3}$ & $(+)^{2}$ & ${ }_{(+)}^{1}$ & & \\
\hline$=1$ & 1 & 1 & 1 & 1 & 1 & 1 & 1 & 1 & 1 & $=1^{Z}$ & \\
\hline & $(-)^{1}$ & & & & & $(-)^{1}$ & & $(-)^{2}$ & & & \\
\hline$=1^{Z}$ & 0 & & & & & 0 & $=1^{Z}$ & 0 & & & \\
\hline
\end{tabular}

Последовательный алгоритм (ПА)

ПА предсказывает, что выпадут L-1эл, равных зондовому элу (Z), а эл в позиции L будет инверсным (пример в таблице 1 , строки 1 , $(\mathrm{Z}=1) 11110 »)$. Инверсный эл предсказывается и учитывается в статистике вместе с элами составного события. Зондовое событие $\mathbf{Z}$ задаёт значения для серии искомых эл и не учитывается в статистике. Поскольку ПА не работает с геометрическими свойствами бинарной пос-ти, то при перемещении в новую зондовую позицию ПА просматривает содержание всех эл на своём пути. При обнаружении эла начала пятого по счёту составного события (число пропускаемых составных событий берётся любым), этот эл объявляется новым Z - элом, и начинает работу алгоритм предсказания ПА.

Разберём работу ПА на ситуациях раздела «Последовательный (ПА)» таблицы 1. В которой рассмотрены некоторые ситуации при угадывании серий с числом эл: $\mathrm{L}=5$. Постулируем, что угаданная серия из пяти эл $(\mathrm{L}=5)$ должна выглядеть в зависимости от значения зондового события $\mathrm{Z}$; для « $\mathrm{Z}=1 »$, так: «11110» (показано в таблице), а для « $\mathrm{Z}=0 »$, так: «00001». Угаданное значение (успешное предсказание) в таблице обозначается знаком «+», не успешное знаком «-».

В разделе «Последовательный (ПА)» строки № 1 таблицы 1 величина зондового эла равна «1» $(\mathbf{Z = 1})$, столбец 1. Так как $\mathbf{Z}=« 1 »$ и $\mathrm{L}=5$, то ПА предсказывает и ищет выпадение цепочки: «11110». В столбце 2 ПА сделал успешное предсказание, рядом с номером предсказания 1 стоит знак «+». Второе, третье, четвёртое предсказания успешны: 2(+); 3(+); 4(+). Пятым предсказанием - столбец 6, ПА предсказывает выпадение 
нуля «0». Оно сбылось $5(+)$. После обнаружения «0», ПА завершает работу в данной области Z, увеличивает счётчик правильно предсказанных цепочек на 1 , перемещается в новую зондовую область Z, пропуская пять составных событий.

В строках № 2 ПА ищет цепочку «11110», так как $(\mathbf{Z}=\mathbf{1})$, столбец 1. Ситуация с предсказаниями повторилась до столбца 6 (позиция $\mathrm{L}=5$, после $\mathrm{Z}$ ), в котором вместо «0» выпала «1». ПА увеличивает счётчик ошибочных предсказаний (-) эл на 1. ПА завершает работу с зондовой областью, увеличивает счётчик не угаданных цепочек на 1, перемещается в следующую зондовую область, пропуская пять составных событий.

В строках № 3 ПА снова ищет цепочку «11110», так как $(\mathbf{Z}=\mathbf{1})$, столбец 1. В столбце 2 при первом же предсказании произошла ошибка предсказания. Вместо предсказанной «1» выпал «0». ПА увеличивает счётчик ошибочных предсказаний (-) эл на единицу. ПА завершает работу с зондовой областью, увеличивает счётчик не угаданных цепочек на 1. Перемещается в следующую зондовую область, ища начало пятого по счёту составного события.

И т. д. по 800000-ое зондовое исследование (для данного эксперимента).

\section{Геометрический алгоритм (ГА)}

Для вызова геометрических свойств в случайной бинарной пос-ти геометрический алгоритм, в отличие от ПА, отсчитывает элы между зондовыми событиями Z (ПА отсчитывает составные события между Z).

После внедрения зонда Z, ГА сперва работает с элами, расположенными до зондового события Z, а затем с элами после события Z. ГА строит свои предсказания так, что предсказывает составное событие из указанного числа эл L, идентичных зондовому элу. Цепочка из L эл ограниченна на своих концах инверсными элами (в таблице 1 , строки 1 , цепочка из $\mathrm{L}=5$ эл, $\mathrm{Z}=\ll 1 », ~ « 01110 »)$. Для $\mathrm{Z}=« 0$ » искомая цепочка длиной в пять эл $(\mathrm{L}=5)$ выглядит так: «10001». ГА предсказывает элы составного события и инверсные элы в первой и последней позиция $\left(\mathrm{L}_{1} \neq \mathrm{Z}\right.$ и $\left.\mathrm{L}_{5} \neq \mathrm{Z}\right)$, результаты предсказания учитываются в статистике. Зондовое событие $\mathbf{Z}$ задаёт предсказываемые значения эл, но не учитывается в статистике.

Разберём работу ГА на ситуациях раздела «Геометрический (ГА)», таблицы 1. Полностью угаданная серия из пяти элементарных событий $(\mathrm{L}=5)$ должна выглядеть в зависимости от значения зондового события так: «01110» или так: «10001». Угаданное значение (успешное предсказание) обозначается «+», не успешное «-».

Строке № 1, столбец 11, зондовый эл равен «1» $(\mathbf{Z}=\mathbf{1})$. Так как $\mathbf{Z}=« \mathbf{1}$ и $\mathrm{L}=5$, то ГА предсказывает выпадение цепочки: «01110».

ГА предсказывает слева до зонда (столбец 10), выпадение события идентичному Z, угадывает (+). ГА повторяет удачные предсказания (столбцы 9, 8). После угадывания в столбце 8, ГА должен предсказать «0», так как он ищет «01110» и ожидает выпадение «0» слева. В столбце 7 показано, что предсказание ГА сбылось. После этого ГА предсказывает выпадение «0» справа (столбец 12). Обнаружив «0» справа (столбец 12), ГА завершает работу с данной областью $\mathrm{Z}$, увеличив на один счётчик угаданных цепочек. Переходит в следующую позицию $\mathbf{Z}$ на 25 эл.

В строках № 2 ГА ищет цепочку «01110», так как $(\mathbf{Z}=\mathbf{1})$, столбец 11. Ситуация с предсказаниями повторялась до столбца 7. В котором вместо «0» выпала «1» - ошибка предсказания. ГА увеличивает счётчик ошибок эл на 1 , завершает работу с областью, увеличивает счётчик ошибок цепочек на 1 , идёт в следующую зондовую область на 25 эл от $\mathbf{Z}$.

В строках № 3 ГА ищет цепочку «01110», так как $(\mathbf{Z}=\mathbf{1})$, столбец 8. В столбце 7 ГА не угадал, вместо «1» выпал «0». ГА воспринимает «0» левым краем искомой цепочки «01110», начинает предсказывать события справа от ZZ. В столбце 9 ГА не угадал, вместо «1» выпал «0». Длина цепочки («01110») не достигнута. ГА

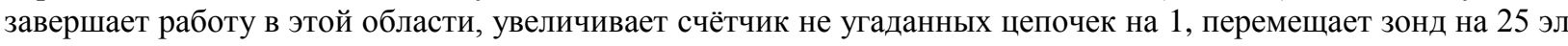
от $\mathbf{Z}$.

Результаты поиска в случайной бинарной пос-ти геометрическим и последовательным алгоритмом приведены в таблице 2 .

Каждый алгоритм сделал по 800000 серий предсказаний. Для адекватного сравнения предсказательной силы алгоритмов в каждой строке таблицы 2 представлены значения, связанные с искомыми сериями одинаковой длины $n$ в элах. Длина искомой серии $n$, в элах дана в столбце 1 . Для поиска каждой серии требуемой длины $n$ алгоритмы запускались на исполнение отдельно. Сумма серий по каждому столбцу: 2.1 и 3.1, равна $8 \cdot 10^{5}$. В столбцах 2.1 и 3.1 даны численности угаданных алгоритмами серий длины n. Число угаданных серий геометрическим алгоритмом обозначается: ${ }^{n} S_{\mathrm{g}}$. Число угаданных серий последовательным алгоритмом обозначается: ${ }^{n} S_{p}$. Поскольку геометрический алгоритм не может искать серии меньше, чем из двух эл ${ }^{n=2} S_{\text {g }}$ (таблица № 1, строки № 3), то в таблице 2 графы для длины в 1 эл, для геометрического алгоритма пропущены.

В столбцах 2.2 и 3.2 таблицы 2 дана численность всех эл, на которые были сделаны алгоритмами предсказания для угадывания искомых цепочек. Столбец $2.2, E l\left({ }^{n} S_{\mathrm{g}}\right)$ - численность эл, на которые сделал ставки (предсказывал) геометрический алгоритм. Столбец $3.2, E l\left({ }^{n} S_{p}\right)$ - численность эл, на которые делал ставки (предсказывал) последовательный алгоритм.

В столбцах 2.3 и 3.3 таблицы 2 рассчитано, сколько в среднем было сделано предсказаний алгоритмом, на одну обнаруженную серию событий заданной длины $n$. 
В столбцах 2.4 и 3.4 таблицы 2 находятся величины обратные, для наглядности восприятия вероятностям нахождения искомых серий. Кривые вероятностей нахождения серий одинаковой длины, геометрическим и последовательными алгоритмами представлены на графике 2.

В столбце 4 показано, во сколько раз вероятность (поисковая мощь) обнаружения серий элементарных событий одинаковой длины больше геометрическим алгоритмом, чем последовательным (смотри график 2).

Таблица 2. Результаты поиска в случайной бинарной последовательности; $N=2 \cdot 10^{7}$

\begin{tabular}{|c|c|c|c|c|c|c|c|c|c|c|}
\hline & \multicolumn{4}{|c|}{ «Геометрический» поиск } & \multicolumn{5}{|c|}{ «Последовательный» поиск } & \multirow{2}{*}{$\frac{(2.4)}{\frac{(3.4)}{P_{\mathrm{g}}^{A}}}$} \\
\hline$e^{n}$ & ${ }^{n} S_{\mathrm{g}}$ & $E l\left({ }^{n} S_{\mathrm{g}}\right)$ & $\frac{E l\left({ }^{n} S_{\mathrm{g}}\right)}{{ }^{n} S_{\mathrm{g}}}$ & $\frac{1}{P_{\mathrm{g}}^{A}}=\frac{Z_{\mathrm{g}}}{{ }^{n} S_{\mathrm{g}}}$ & ${ }^{n} S_{p}$ & $E l\left({ }^{n} S_{p}\right)$ & $\frac{E l\left({ }^{n} S_{p}\right)}{{ }^{n} S_{p}}$ & $\frac{1}{P_{p}}=$ & $=\frac{Z_{P}}{{ }^{n} S_{p}}$ & \\
\hline 1 & 2.1 & 2.2 & 2.3 & 2.4 & 3.1 & 3.2 & 3.3 & & 3.4 & 4 \\
\hline el & нет & нет & нет & нет & $5^{399}$ & $00^{8000}$ & 2,00 & & 2,00 & нет \\
\hline el 2 & $766^{200}$ & $711^{1200}$ & 5,98 & 3,98 & $2^{200}$ & $806^{1199}$ & 5,99 & & 4,00 & 1 \\
\hline $\mathrm{el}^{3}$ & $590^{200}$ & $890^{1999}$ & 9,97 & 3,99 & $52^{996}$ & $196^{1399}$ & 14,0 & & 8,03 & 2 \\
\hline el 4 & $923^{148}$ & $184^{2498}$ & 16,77 & 5,37 & $15^{499}$ & $533^{1498}$ & 30,0 & & 16,03 & 3 \\
\hline el 5 & $080^{100}$ & $914^{2797}$ & 27,96 & 7,99 & $5^{249}$ & $776^{1549}$ & $\begin{array}{ll} & 62,1 \\
0 & \\
\end{array}$ & & 32,06 & 4 \\
\hline el ${ }^{6}$ & $15^{628}$ & $544^{2972}$ & 47,32 & 12,73 & $14^{125}$ & $752^{1574}$ & $84^{125,}$ & & 64,12 & 5 \\
\hline el $\begin{array}{l} \\
\text { el }\end{array}$ & $32^{376}$ & $796^{3071}$ & 81,63 & 21,25 & $\begin{array}{ll} & 627 \\
1 & \end{array}$ & $465^{1588}$ & $30^{253,}$ & 2 & 127,2 & 6 \\
\hline & $\begin{array}{r}Z_{G} \\
(Z=1) 11\end{array}$ & $=80000$ & $1 \leftarrow Z Z$ & $\rightarrow \ldots \mid ; 01$ & $\begin{array}{c}\mathrm{Z} \\
(\mathrm{Z}=1) 11\end{array}$ & $\begin{array}{r}=800 \\
110\end{array}$ & 000 & $\mathrm{Z}$ & $\rightarrow \ldots$ & \\
\hline
\end{tabular}

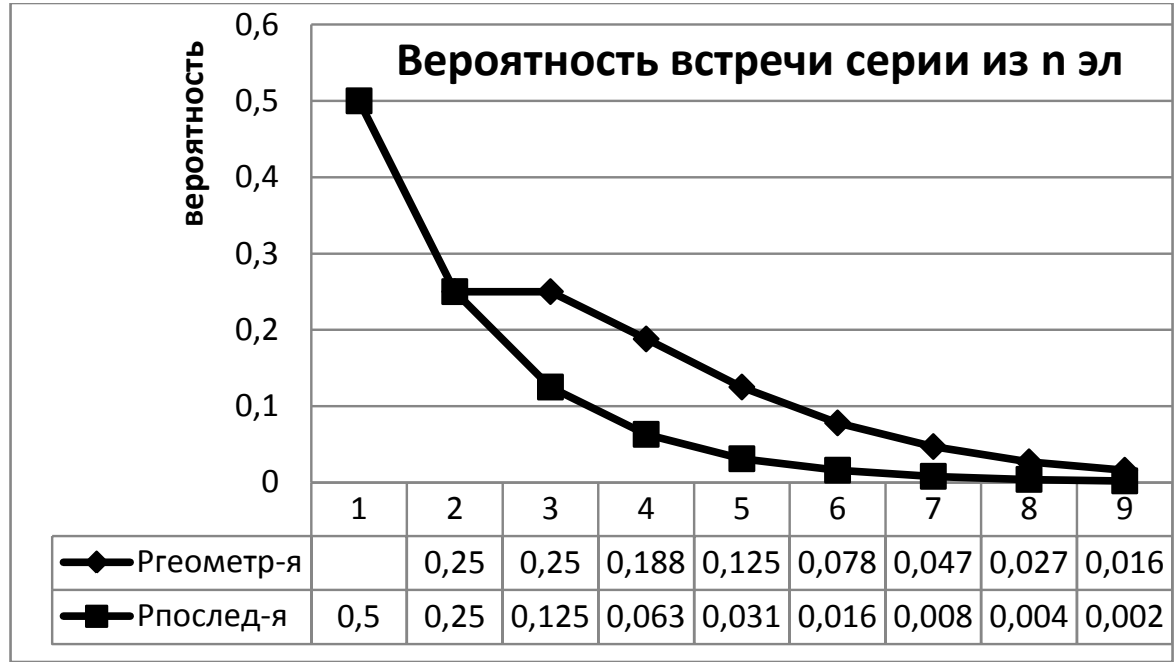

График 2. Вероятность встречи из п эл

Обозначим вероятность, полученную в результате работы геометрического поискового алгоритма ГА, как: $P_{\mathrm{g}}^{A}$..

При помощи таблицы 2 продемонстрируем некоторые формульные зависимости, связывающие её столбцы друг с другом.

Отношение вероятности обнаружения серии из $n$ эл, геометрическим алгоритмом $P_{\mathrm{g}}^{A}$ к последовательному алгоритму $P_{p}$, равно: $n-1$, ф. 3.1 : 


$$
\frac{P_{\mathrm{g}}^{A}}{P_{p}}=n-1
$$

В столбцах $3.4\left(\frac{1}{P_{p}}\right)$ и $2.4\left(\frac{1}{P_{\mathrm{g}}^{A}}\right)$, таблицы 2 даны обратные величины вероятностей, разделив столбец 3.4 на 2.4 получим: $\frac{1}{P_{p}}: \frac{1}{P_{\mathrm{g}}^{A}}=\frac{P_{\mathrm{g}}^{A}}{P_{p}}=n-1$.

Вероятность обнаружения серии потоковым алгоритмом обратно пропорциональна степени двойки и описывается ф. 3.2:

$$
P_{p}=\frac{1}{2^{n}} ; n=1,2,3, \ldots
$$

Где $n$ - длина искомой серии.

Перепишем ф.3.1 в виде $P_{\mathrm{g}}^{A}=P_{p} \cdot(n-1)$. Раскрывая $P_{p}$ по ф. 3.2 получим $P_{\mathrm{g}}^{A}$ - вероятность обнаружения серии из $n$ эл геометрическим алгоритмом, выраженную через длину $n$ искомой серии, ф. 3.3:

$$
P_{\mathrm{g}}^{A}=P_{p} \cdot(n-1)=\frac{n-1}{2^{n}}
$$

Значения в столбце 2.4 рассчитываются как: $\frac{1}{P_{\mathrm{g}}^{A}}=\frac{2^{n}}{n-1}$..

В столбце 3.4 дан результат деления числа зондовых внедрений $Z_{P}$ на число угаданных цепочек ${ }^{n} S_{p}$ длины $\mathrm{n}$ (столбец 3.1): $\frac{1}{P_{p}}=\frac{Z_{P}}{{ }^{n_{p}}}=2^{n}$. Так как: $P_{p}=\frac{1}{2^{n}}$, то число угаданных цепочек ${ }^{n} S_{p}$ при числе внедрений зонда $Z_{P}$, будет рассчитываться по ф.3.4:

$$
{ }^{n} S_{p}=P_{p} \cdot Z_{P}
$$

Число найденных последовательным алгоритмом серий ${ }^{n} S_{p}$, столбец 3.1 , связанно с числом всех серий $Z_{P}$ (в которых пытались найти серию нужной длины), по ф. 3.5:

$$
{ }^{n} S_{p}=\frac{Z_{P}}{2^{n}}
$$

В столбцах 2.3 и 3.3 показаны рассчитанные по экспериментальным данным средние числа эл, приходящиеся на одну угаданную серию. Для последовательного поискового алгоритма (столбец 3.3 ) число эл приходящихся на одну серию рассчитывается по ф. 3.6:

$$
\frac{E l\left({ }^{n} S_{p}\right)}{{ }^{n} S_{p}}=2 \cdot \frac{Z_{P}}{{ }^{n} S_{p}}-2
$$

Подставив ${ }^{n} S_{p}$ из ф. 3.5 в ф. 3.6 получим ф. 3.7 для столбца 3.3 :

$$
\frac{E l\left({ }^{n} S_{p}\right)}{{ }^{n} S_{p}}=2^{n+1}-2
$$

Отсюда, математическое ожидание числа элементарных событий, которые необходимо затратить на нахождение ${ }^{n} S_{p}$ серий при поиске последовательным алгоритмом, рассчитывается по ф. 3.8 (столбец 3.1 ):

$$
E l\left({ }^{n} S_{p}\right)={ }^{n} S_{p} \cdot\left(2^{n+1}-2\right)=\frac{Z_{P}}{2^{n}} \cdot\left(2^{n+1}-2\right)
$$

Где $n$ - длина искомой серии. 
Число событий ${ }^{n} S_{G}$ (столбец 2.1 таблицы 2), найденных по геометрическому алгоритму рассчитывается по ф.1.2. Отметим, что вероятность попасть в составное событие длины n описывается ф. 1.1:

${ }^{n} p_{g}=\frac{n}{2^{n+1}} ; n_{i}=1,2,3, \ldots$, а вероятность найти с помощью геометрического алгоритма серию из $\mathrm{n}$ эл описывается ф. 3.3: $P_{\mathrm{g}}^{A}=\frac{k-1}{2^{k}} ; k_{i}=2,3,4, \ldots$, Значения этих вероятностей совпадут при сдвиге их нумерации на единицу: ${ }^{n} p_{g}(i)=P_{\mathrm{g}}^{A}(i+1) ; i=1,2,3, \ldots$. .. Этот сдвиг отражает то, что стартовая длина поисковой серии для алгоритма равна двум (невозможно алгоритмом обнаружить единичное событие, вероятность равна нулю), в то время как геометрическая вероятность попасть в единичное событие равна 0,25 .

Обсуждение

Так как при одинаковом количестве попыток найти заданные серии одинаковой длины, геометрический алгоритм находит больше серий, чем последовательный, то эти алгоритмы имеют разную поисковую мощь. Можно выдвинуть гипотезу, что различие поисковой мощи у алгоритмов определяется различием в поисковых степенях свободы. Чем больше степеней поисковой свободы, тем большей мощью обладает поисковый алгоритм.

Последовательный поисковый алгоритм обладает одной степенью свободы, он может искать только справа от зонда.

Геометрический поисковый алгоритм обладает двумя степенями поисковой свободы, он ищет слева и справа от зонда.

Для увеличения вероятности нахождения искомых серий алгоритм геометрического поиска требует существования не рассматриваемых элементарных событий, которые пропускаются алгоритмом без определения их содержимого («0» или «1»). Не рассматриваемые элы можно сравнить со своеобразной «тёмной материей» вероятностного пространства. «Тёмная материя» из эл принципиально должна существовать для работы геометрического алгоритма.

Материя иерархична. Отдельные выпадения монеты, собранные по Мизесу в коллективы, приобретают новые групповые свойства. Сохраняя непредсказуемость для одной монеты, коллективы получают свойство предсказуемости для серий выпадений монет. Установлены законы, выведены формулы, позволяющие по выпавшим сериям управлять выпадениями будущих серий $[6,7]$ через механизм отказа от рассмотрения выпадающих событий (на практике это значит не делать ставок несколько игр подряд).

При одинаковом количестве поисковых испытаний (для каждого рассмотренного алгоритма 800000 ), геометрический алгоритм демонстрирует уверенное преимущество по числу найденных серий: ${ }^{n} S_{G}>$ ${ }^{n} S_{p}$; при $n>2$, столбцы 2.1 и 3.1 таблицы 2.

На самом деле, привлечение геометрического объяснения является не обязательным. Эта статья другими словами описывает процесс, который объясняется без геометрических пространственных построений [2, 5, 7]. Подключение пространственных построений связано с надеждой, что наглядность улучшит понимание физического процесса регулировки вероятностей для выпадения серий монеты, и с тем, что геометрическая вероятность хорошо освоена в военных применениях (стрельба по мишеням), и её существование не вызывает сомнений.

Вместо описанной геометрической интерпретации можно было предложить типичный эксперимент с экстрасенсом на угадывания. Когда для экстрасенса готовиться цепочка монет, накрытых сверху непрозрачными карточками (или сосудами). Случайность значений монет («0», «1») подтверждается высоконаучной комиссией. Экстрасенс перемещается вдоль накрытых монет, указывает на накрытую монету и заявляет (предсказывает), что будет под ней («0», «1»). Если он применит методику, описанную в работах $[2,5,7]$ или в этой статье, то высоконаучная комиссия зафиксирует его необычные экстрасенсорные способности. Так как он продемонстрирует повышенные результаты угадываний называемых им серий, по сравнению с полученной на основе рассуждений о равновероятностных исходах формулой: $\frac{1}{2^{n}}$. В случае, когда случайная бинарная пос-ть лежит на столе под карточками, можно угадать больше серий, чем в случае предсказывания результатов выпадения монеты, с последующим подбрасыванием монеты.

Перемещение экстрасенса вдоль ряда с карточками даёт ему возможность работы с геометрическими вероятностями. Но эти же перемещения можно сравнить и с перемещением во времени.

Интересно понять следующую модель. Ряд карточек закрывает события - пос-ть выпадений стороны одной монеты. Перемещение над пос-тью событий одного и того же предмета можно осуществлять только во времени. Таким образом, перемещение над рядом из событий пос-го выпадения одной монеты, накрытых карточками, является моделью перемещения во времени. Но одновременно это же перемещение является и геометрической вероятностью. В данной модели перемещения над карточками время и пространство объединяются в одно.

Первая система игры - удвоение ставки, известна уже более 100 лет. Игра Пенни - вторая «системы игры» в мизесовском смысле (Мизес умер в 1953 г.) гарантирует выигрыш игрока в орлянку. Её правила были опубликованы в октябре 1969 года в журнале «Journal of Recreational Mathematics». В России 50 лет игнорируется существование «системы игры» Пенни.

\section{Выводы}

В статье представлена ёщё одна система игры (в мизесовском смысле). Для получения двух численно разных вероятностей при обнаружении серий выпадений монеты равной длины была успешно 
задействована геометрическая вероятность. Получены две формулы вероятностей обнаружения серий равной длины: ф. 3.3 для вероятности обнаружения геометрическим алгоритмом поиска и ф. 3.2 для вероятности обнаружения последовательным алгоритмом поиска.

Вероятность обнаружения серий одинаковой длины в случайной бинарной последовательности зависит от правил поиска этих серий. В статье показано, что правила игры в мизесовском смысле (управление вероятностью выпадений серий случайных событий) существуют.

Показано, что результаты угадываний зависят от фактора времени (свершённости события). А именно, в зависимости от того: делаются ли предсказания на уже выпавших монетах, с неизвестным результатом выпадения «0», «1» (к каждой из выпавших монет возможен доступ), или предсказание делается для одной монеты на её будущее выпадение (после каждого предсказания монета подбрасывается для проверки правильности сделанного предсказания).

Пространственный процесс перемещения вдоль ряда выпавших бинарных событий, но с их неизвестным содержанием является моделью перемещения во времени. В статье даны экспериментально найденные формулы для процессов, меняющих вероятность обнаружения серий выпавших событий. Следовательно, в мире, в котором возможно перемещение во времени, действуют формулы вероятности из этой статьи, и в таком мире есть возможность менять вероятность обнаружения серий событий (менять условия для развития процессов на постоянном множестве элементарных событий).

\section{Лuтература}

1. Филатов О. В., Филатов И. О., Макеева Л. Л. и др. «Потоковая теория: из сайта в книгу». Москва, «Век информации», 2014. С. 200.

2. Филатов О.В., Филатов И. О. «Закономерность в выпадении монет - закон потоковой последовательности». Германия, Издательский Дом: LAPLAMBERT Academic Publishing, 2015, c. 268.

3. Филатов О. В., Филатов И. О. Статья «О закономерностях структуры бинарной последовательности», «Журнал научных публикаций аспирантов и докторантов», № 5, 2014.

4. Филатов О.В. Статья «Теорема «О амплитудно-частотной характеристике идеальной бинарной случайной последовательности», «Проблемы современной науки и образования», № 1 (31), 2015 г.

5. Филатов О.В. Филатов И. О. Статья «О закономерностях структуры бинарной последовательности (продолжение)», «Журнал научных публикаций аспирантов и докторантов», № 6, 2014.

6. Филатов O. В. Статья «Расчёт численностей поисковых шаблонов в парадоксе Пенни», «Проблемы современной науки и образования», № 11 (41), 2015 г.

7. Филатов О.В. Статья «Описание схем управления вероятностью выпадения независимых составных событий», «Проблемы современной науки и образования», № 2 (44), 2016 г. 